\title{
Mechanical properties of concrete to cyclic uniaxial tensile loading using variable waveforms
}

\author{
YUZHI $\mathrm{CHEN}^{1,2}, \mathrm{XUDONG} \mathrm{CHEN}^{2, *}$ and JINGWU BU ${ }^{3}$ \\ ${ }^{1}$ Architectural Engineering Institute, Jinling Institute of Technology, Nanjing 211169, China \\ ${ }^{2}$ College of Civil and Transportation Engineering, Hobai University, Nanjing 210098, China \\ ${ }^{3}$ College of Water Conservancy and Hydropower Engineering, Hohai University, Nanjing 210098, China \\ e-mail: cxdong1985@hotmail.com; cxdong1985@163.com
}

MS received 2 March 2016; revised 20 June 2016; accepted 3 July 2016

\begin{abstract}
The mechanical properties of concrete under cyclic tensile loading using square waveform, sine waveform and ramp waveform are studied. The experiments are performed on a closed-loop electro-hydraulic servo-controlled material testing system (MTS). The axial strain, dissipated energy per loading cycle, the damage evolution law and deformation modulus are mainly studied. The results show that the three-stage evolution law of axial strain and damage variable of concrete under ramp waveform and sine waveform are more obvious than those under the square waveform. The dissipated energy changes at different stages of fatigue life. At the beginning and end of the fatigue life, the rate of dissipated energy is higher than that at the medium stage of the fatigue time, which is attributed to the formation of cracks. The evolution of deformation modulus of concrete subjected to cyclic tensile loading using three loading waveforms also shows three stages: fast increase in the damage — increase at a slow constant rate—and accelerated increase in damage until failure.
\end{abstract}

Keywords. Cyclic; uniaxial tension; dissipated energy; damage evolution; waveform; concrete.

\section{Introduction}

Concrete structures in civil engineering are vulnerable to dynamic loading, such as explosion, impacting and earthquakes. The mechanical properties of concrete under dynamic loading are related to those under static loading but different from them. For example, strength of concrete under cyclic loading is lower than the static strength under monotonic loading. The mechanical behaviour of concrete structure subjected to repetition of loading is called fatigue. The mechanical properties and damage evolution of concrete under cyclic loading are still hot research areas in civil engineering.

Numerous experiments on mechanical properties of brittle materials under cyclic loading have been conducted and some achievements have been obtained. The behaviour of rocks under cyclic loading has been studied using one waveform [1, 2]. In reference [3] the authors investigated the fatigue deformation properties and fracture mechanisms of sandstone, marble and granite under uniaxial compression by systematic experiments. The evolution of axial strain can be divided into three stages, i.e., initial deformation stage, deformation increases at a constant speed and accelerated deformation stage. In the whole process, the deformation accumulation leads to final failure of rocks.

*For correspondence
The effect of lateral pressure on hysteresis evolution of sandstone under fatigue loading was studied in reference [4]. It found that with increasing lateral pressure, the area of hysteresis loop, i.e., dissipated energy per loading cycle, increased. Some researchers [5] used two loading waveforms and two control modes to conduct cyclic tests. In each cycle, the deformation caused by sine waveform loading is larger than that by triangle waveform loading. The larger the cycle amplitude, the shorter the working life. Some experimental studies on the fatigue properties of brittle materials $[6,7]$ have demonstrated that both loading waveform and amplitude are of great significance and affect the rock behaviour. Damage is accumulated most rapidly under square waveforms with a high energy requirement. A ramp waveform produced the least damaging of those considered. The dynamic effect and influence on the mechanical properties of brittle material under three different waveforms have been analysed theoretically in reference [8]. The authors found that the tensile strength and strain rate under sine waveform are far less than those under square waveform but a little more than that under ramp waveform, that is to say, the dynamic effect under sine waveform is worse than under square waveform but better than under ramp waveform when the loading frequency and amplitude maintain given values. Moreover, the dynamic effect enhanced with the increase of loading frequency and amplitude under a certain waveform. Medeiros 
et al [9] and Saucedo et al [10] found that the loading frequency effect on the fatigue behaviour of plain concrete is pronounced. The fatigue life at lower frequencies is less than that at higher frequencies.

Fatigue is progressive damage to material that occurs due to repeated loading. Developing a statistically reliable model to predict fatigue life has always been a major area of interest for researchers. Many fatigue life models that relate the fatigue life of the structure to stress level have been proposed based on fatigue test results. The Wholer equation based on fatigue test results conducted by Kesler [11] and Raithby and Galloway [12] was calibrated in reference [13]. In the developed model, fatigue failure is defined as the complete fracture of a concrete beam. In reference [14], the effect of the stress ratio (ratio of minimum to maximum stress level) on fatigue behaviour of concrete was first investigated. Since then, many researchers [15, 16] have adopted a relationship that incorporates the effect of stress ratio on fatigue life of concrete. The concept of equivalent fatigue life was introduced in reference [17] to incorporate the stress ratio into fatigue life model. In reference [18], the authors calibrated McCall's formula for hybrid fibrous concrete and produced a group of $S-N-P_{\mathrm{f}}$ curves for different combinations of polypropylene- and steel-fibre-reinforced concrete. The energy absorption characteristic of concrete in the course of fatigue life has long been an area of interest for researchers. The energy absorption capacity of concrete due to its nonlinear hysteresis behaviour under cyclic loading has been reported in many studies [19, 20]. It demonstrated that average dissipated energy per loading cycle is a good estimator of fatigue life for fibre-reinforced concrete. The model based on the dissipated energy approach has greater predictive quality compared with the traditional fatigue life model (i.e., $S-N_{\mathrm{f}}$ curve). Reference [21] proposed mathematical models to evaluate the residual strength of concrete under fatigue loading using linear elastic and nonlinear fracture mechanics properties. It is observed that the predicted remaining life using power model and modified bilinear model is in good agreement with the corresponding experimental values.

The existing research on concrete fatigue behaviour is focused on the compressive or flexural loading. However, the tensile mechanical properties of brittle materials like concrete are significant to structure engineering safety. Recently, some researchers have acquired a better test method to study the tensile mechanical properties of concrete [22-26]. But the fatigue behaviour of concrete under tensile loading has not been studied. Hence the fatigue mechanical behaviour of concrete under cyclic uniaxial tensile loading deserves research.

The objective of this paper is to study the mechanical properties of concrete subjected to cyclic direct tension using three variable loading waveforms including square, sinusoidal and ramp waveforms. Both the evolution law of axial strain and the dissipated energy per loading cycle of concrete under cyclic loading are investigated by the authors. Besides, the damage evolution and deformation modulus of concrete along with cyclic loading are analysed in this paper.

\section{Experimental program}

The test is conducted on an electro-hydraulic servo-controlled material testing system (MTS 322). The longitudinal strain is tested by using the MTS extensometers. C40 normal concrete is used in this experiment. The diameter of the cylindrical specimen is $74 \mathrm{~mm}$ and the height is $150 \mathrm{~mm}$. After casting, the specimens are cured in water for 60 days. The ends of specimens are cemented with steel discs by structural adhesive, and the steel discs are connected with test machine by spherical joints and screws. The test machine applies force on the specimen by gripping the screws of spherical joints, which can reduce eccentricity. Three extensometers are arranged around the specimen and the strain of specimen is calculated by averaging the three extensometer values. The equipped specimen is shown in figure 1.

Square, sinusoidal and ramp waveforms are used in this experiment. Load control mode is selected and the loading frequency is $2 \mathrm{~Hz}$. The stress level (the ratio of maximum loading stress to the ultimate strength in tension) is 0.95 . Parameters $P, I$ and $D$ of the test machine are debugged to make the actual waveform basically correspond with the set one.

\section{Test results}

\subsection{Stress-strain curve}

As the waveforms differ, the time to failure of specimens is also different. The number of load repetitions $N$ that causes fatigue failure is the least (36 cycles) when the square waveform is used. For the sinusoidal waveform, the total number of cycles to failure is 175 . The time to failure of

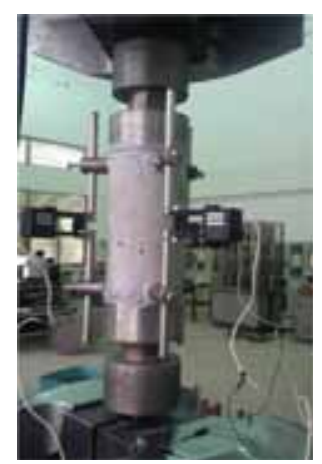

Figure 1. Instrumented concrete specimen under direct tensile loading. 

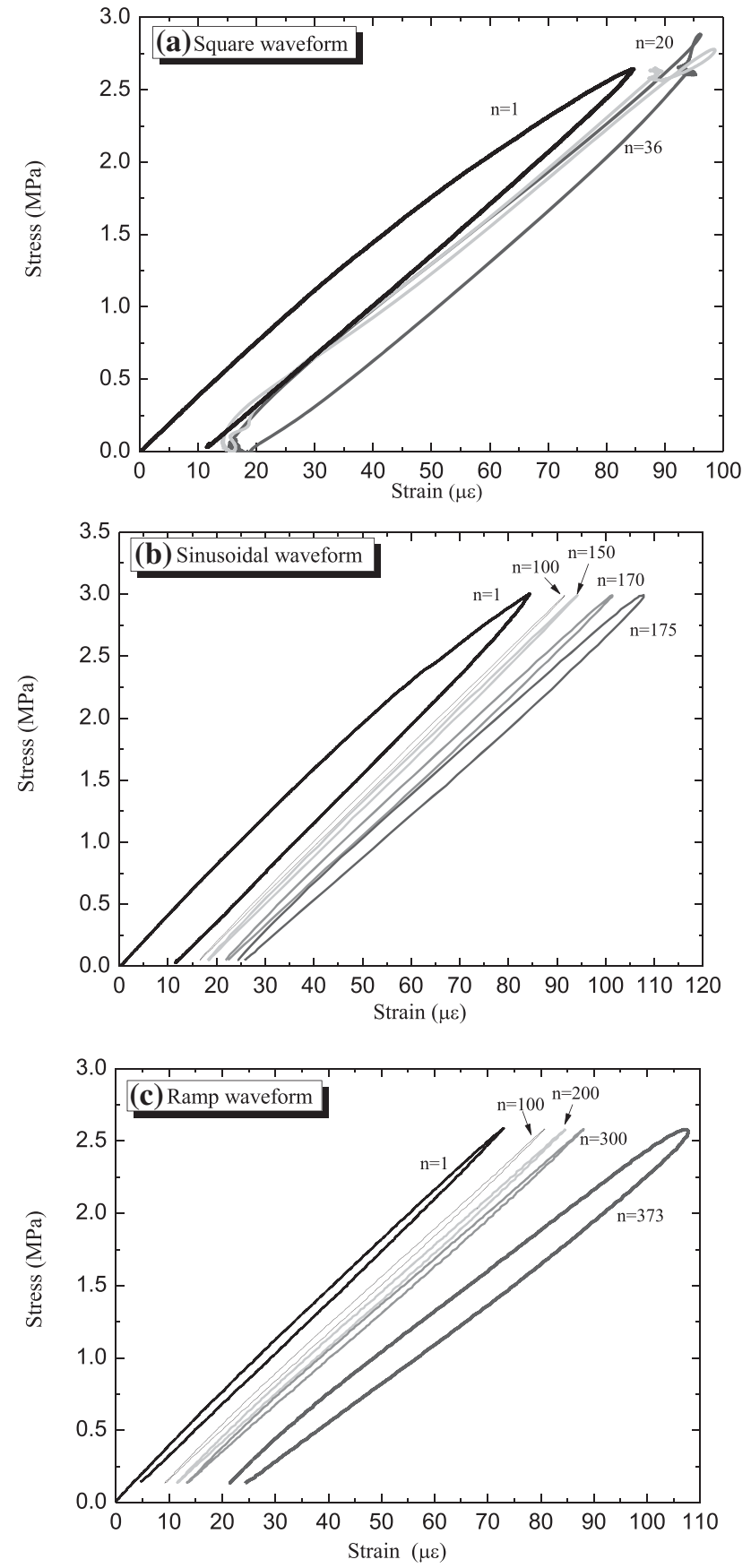

Figure 2. Stress-strain relations of concrete subjected to cyclic loading in three waveforms.

specimen is the longest when ramp waveform is used, and it is 373 cycles.

Figure 2 shows the stress-strain curves of concrete subjected to cyclic loading in tension under three variable waveforms. In figure $2, n$ represents the number of repetitions. It can be seen that the unloading curve cannot coincide with the loading curve for all cycles. The axial strain cannot recover completely and hysteresis is formed since concrete shows elasto-plastic response under cyclic loading.

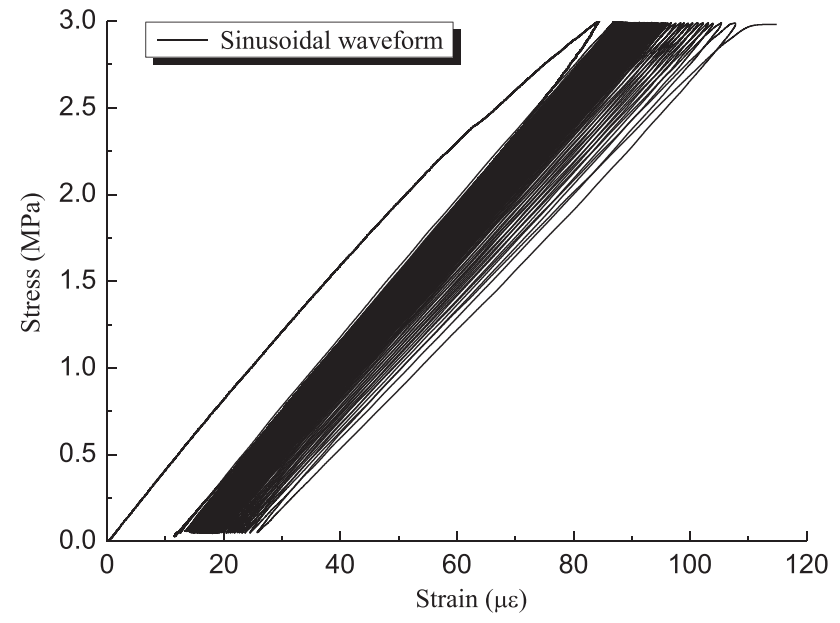

Figure 3. Stress-strain curves under cyclic loading with sinusoidal waveform.

\subsection{Axial strain}

Figure 3 shows the stress-strain curve of concrete subjected to cyclic loading in sinusoidal waveform. At the initial loading stage, the loops of stress-strain curve are sparse. With the increase of the number of repetitions, the stress-strain curve moves towards the direction of increasing strain and becomes increasingly intensive. And when it approaches failure, the hysteresis becomes sparse again. The hysteresis loops represent sparse-intensivesparse in the whole loading process.

Figure 4 shows a partially enlarged detail of stress-strain curve of concrete under cyclic loading in sinusoidal waveform. It can be found that the fatigue process of concrete can be categorized to three stages: initial fast increase stage, constant speed increase stage and accelerated increase stage. In the initial stage, residual strain accumulates fast. In the second stage, the residual strain accumulates at a fairly lower constant speed, but the stage is longer, holding almost $80 \%$ of the whole fatigue process. When the fatigue process approaches the accelerated stage, with the increase of axial strain, the stress and strain also increase and the strain rate increases rapidly, which leads to specimen failures. The third stage is transient and the number of repetitions is less.

Figure 5 shows the axial strain versus cycle ratio $(n / N)$ curve of concrete under cyclic loading in variable waveforms. With the increase of normalized cycle ratio, the axial strain of concrete increases. The growth tendency of axial strain accords with the three-stage evolution form. In the initial stage, axial strain increases rapidly with the increasing of cycle ratio. In the second stage, the axial strain accumulates at a fairly low speed, which is a longer process. The third stage is a transient rupture process, in which the axial strain increases suddenly. In the case of three variable waveforms, the ramp waveform produces the most obvious three-stage damage evolution. 

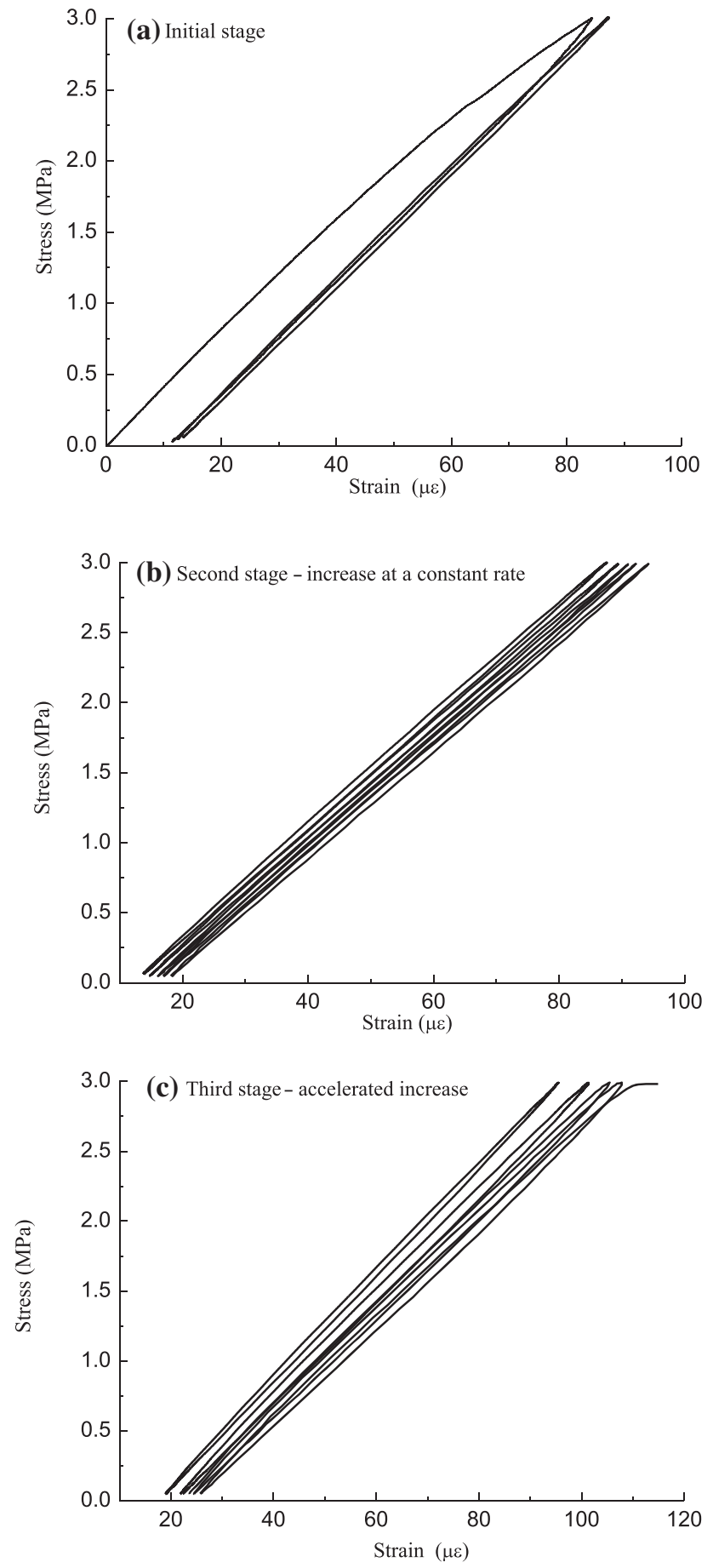

Figure 4. Partially enlarged detail of stress-strain curve of concrete under cyclic loading with sinusoidal waveform.

It can be seen from figure 5 that the accumulation speed of axial strain is the lowest in the second stage. In the whole damage evolution, the second stage is the longest.

Micropores and microcracks exist in the concrete before the force is applied on it. When the force is applied on it, the existing micropores or microcracks extend along the

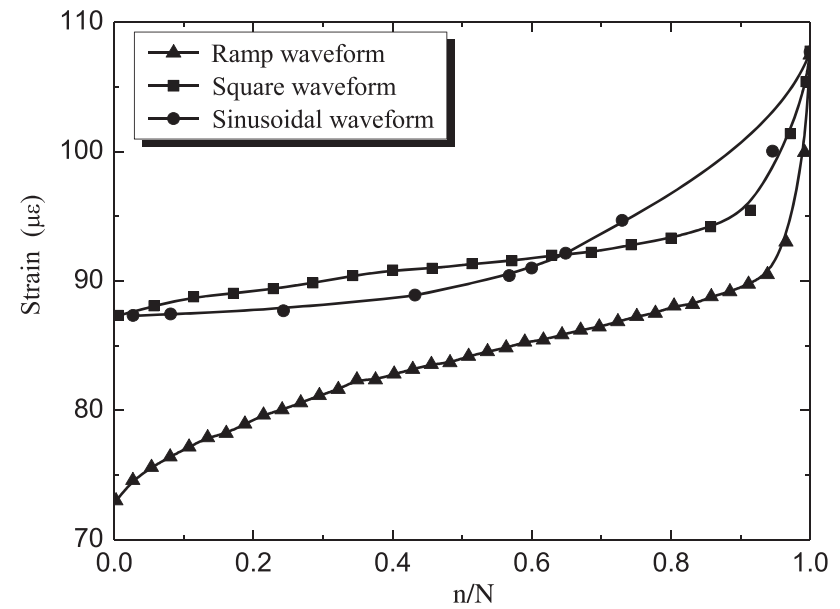

Figure 5. Relation curves of axial strain versus cyclic ratio.

weakest surface in concrete, which forms the strain of specimen. If the extended cracks tend to be parallel with the direction of maximum principal stress, the stress intensity decreases and it leads to stability. By this time, the initial stage is coming to an end. In the second stage, the axial strain accumulates at a lower constant speed which is similar to creep. The creep phenomenon lasts until any strain cannot be permitted to accumulate in concrete itself. At the same time, with the increasing of stress intensity, the propagation and coalescence of internal microcracks lead to the failure of concrete.

\subsection{Energy dissipation in the fatigue process}

Fatigue testing is associated with transferring kinetic energy to energy stored in the body of the sample in the form strain energy. Part of this energy dissipates in other manners. Energy dissipation in the fatigue process can be attributed to three distinctive mechanisms. First, energy dissipates due to the growth of macro-cracks. The second cause of energy dissipation in concrete fatigue is due to material damping. Finally, energy loss due to inter-planar friction, so-called slip damping, is the third mechanism of energy dissipation.

Figure 6 shows the third hysteresis loop of concrete in sinusoidal waveform fatigue loading process. The area underneath the loading portion represents the stored energy in the body of the sample, which is equal to the area of ABDC, denoted as $S_{\text {loading. }}$. Once the load is removed, part of the stored energy transforms to kinetic energy. The area underneath the unloading portion of the stress strain curve represents the released energy, which is equal to the area of BDFE, denoted as $S_{\text {unloading. }}$. The difference between these two areas is the amount of dissipated energy per loading cycle, denoted as $S_{\text {hysteresis }}$. Figure 6 shows the area that is 


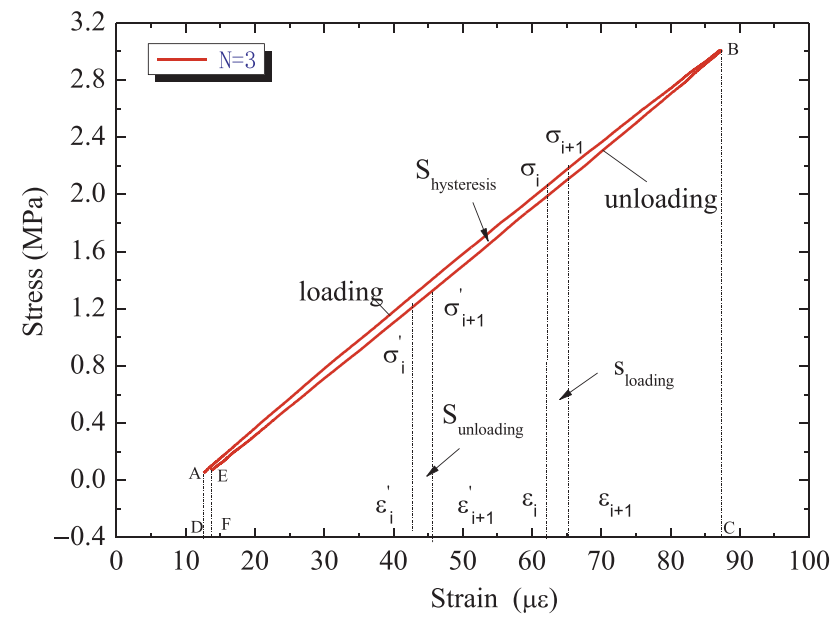

Figure 6. Demonstration of dissipated energy per loading cycle using hysteresis loop.

considered to be dissipated energy per loading cycle, $E_{D}$. In the test, data acquisition is set to record the load and displacement along the loading points at a rate of 200 data points per loading cycle. These data points can be converted to stress and strain values as shown in figure 6 . These data points are used as the input raw data to calculate dissipated energy per cycle. Equation (1) is used to calculate dissipated energy per loading cycle, which is expressed as follows:

$$
\begin{aligned}
S_{\text {hysteresis }}= & S_{\text {loading }}-S_{\text {unloading }} \\
= & \sum_{\text {loading }}\left(\sigma_{i}+\sigma_{i+1}\right)\left(\varepsilon_{i}+\varepsilon_{i+1}\right) / 2 \\
& -\sum_{\text {loading }}\left(\sigma_{i}^{\prime}+\sigma_{i+1}^{\prime}\right)\left(\varepsilon_{i}^{\prime}+\varepsilon_{i+1}^{\prime}\right) / 2 .
\end{aligned}
$$

Figure 7 shows relations of the stored total energy and elastic strain energy versus cycle ratio. It can be seen that the total strain energy with cycle ratio shows decreasestability-increase in three stages. The elastic strain energy increases and shows S-shape three-stage evolution as shown in figure $7 \mathrm{~b}$.

Figure 8 shows the relationship between the dissipated energy per cycle and cycle ratio. The dissipated energy per cycle is the difference between total strain energy and elastic strain energy. It is evident that the amount of dissipated energy changes at different stages of fatigue life. At the beginning and end of the fatigue life, the rate of dissipated energy is higher than that at the medium stage of the fatigue time. In the initial stage of fatigue life, which is commonly called "crack initiation", the load is uniform distributed throughout the concrete. Higher dissipated energy is caused by the formation of initial crack. At the end of fatigue life, the sudden increase in cracks could cause a higher dissipated energy. The medium stage occupies the longest time of fatigue life, in which the microcracks propagate stably.
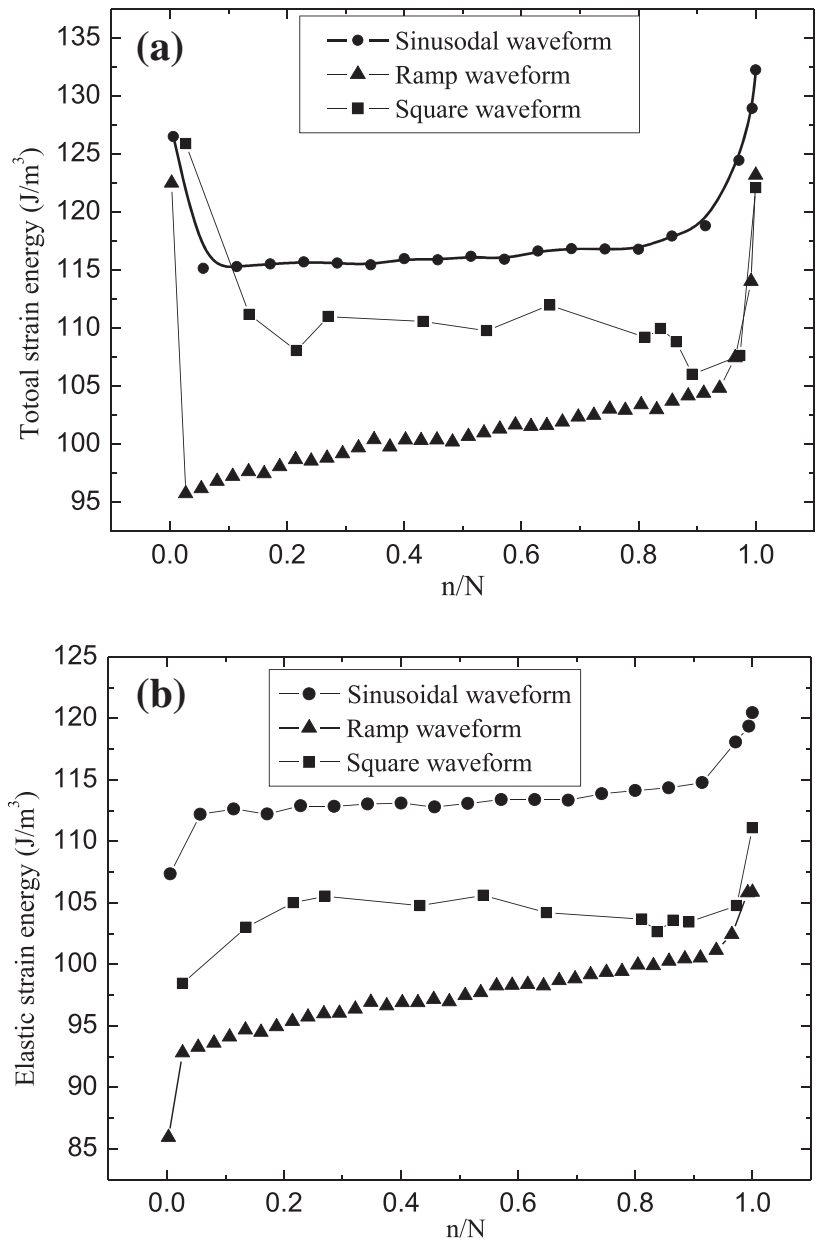

Figure 7. Relations of total strain energy, elastic strain energy versus cyclic ratio under various waveforms.

\subsection{Damage evolution}

Based on continuum damage mechanics, the damage is defined as the microcracks and microvoids of concrete produced by the force applied on it. In the case of one dimension, the damage variable $D \quad(0<D<1)$, describing damage accumulation, can be treated as a scalar quantity.

Based on continuum damage mechanics and elastoplastic damage constitutive model, the damage variable can be expressed as follows [27]:

$$
D=\frac{\varepsilon-\varepsilon_{0}}{\varepsilon_{\mathrm{d}}-\varepsilon_{0}} \frac{\varepsilon_{\mathrm{d}}}{\varepsilon}
$$

where $D$ is the damage variable, $\varepsilon$ is total strain, $\varepsilon_{0}$ is the uniaxial strain in the initial undamaged state and $\varepsilon_{\mathrm{d}}$ is the uniaxial strain in the completely damaged state.

The damage is determined according to Eq. (2) by using the test data. Figure 9 shows the damage variable versus cycle ratio for concrete subjected to fatigue tensile loading in various waveforms. The damage evolution presents the three-stage process, namely, the initial rapid accumulation 


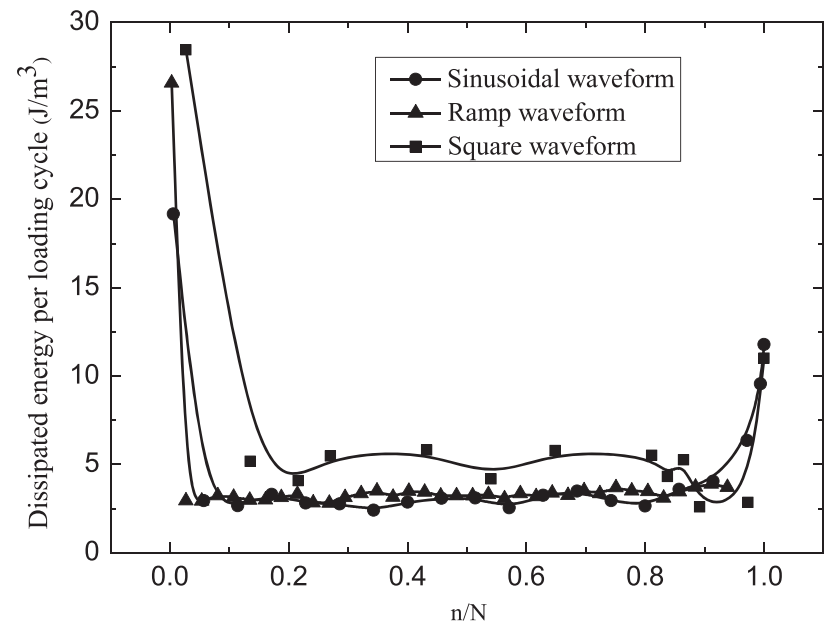

Figure 8. Relations of dissipation energy per loading cycle and cyclic ratio.

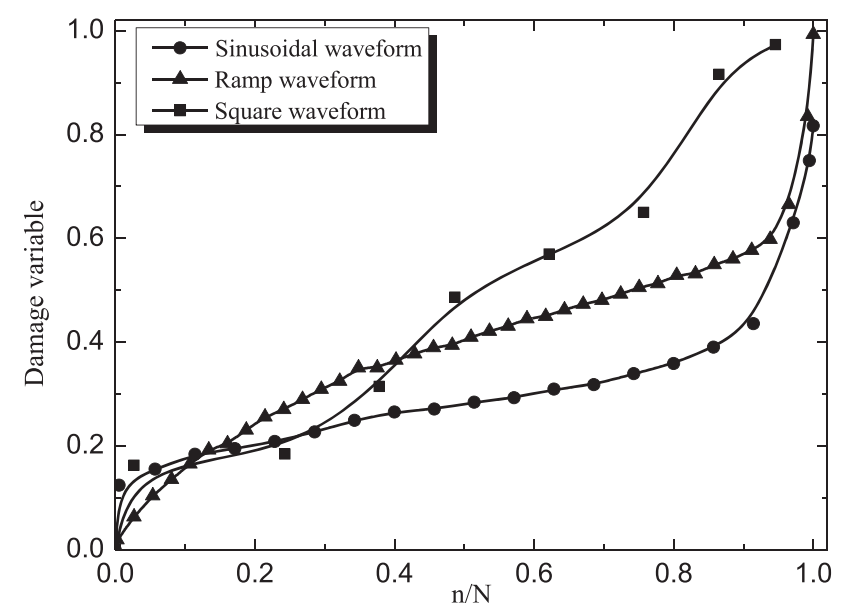

Figure 9. Relations of concrete damage and cyclic ratio under various waveforms.

stage, stable developing stage and accelerated accumulation stage. This character in damage evolution is more obviously followed with sinusoidal and ramp waveforms than the square waveform.

\subsection{Deformation modulus}

The deformation modulus of concrete can be calculated as follows:

$$
E_{d}=\left(\sigma_{\max }-\sigma_{\min }\right) /\left(\varepsilon_{\max }-\varepsilon_{\min }\right)
$$

where $\sigma_{\max }, \sigma_{\min }$ are maximum and minimum axial stress at each cycle, respectively; $\varepsilon_{\max }, \varepsilon_{\min }$ are the corresponding axial strains.

Figure 10 shows the deformation modulus versus cycle ratio for concrete under fatigue tensile loading in various waveforms. The evolution of deformation modulus accords

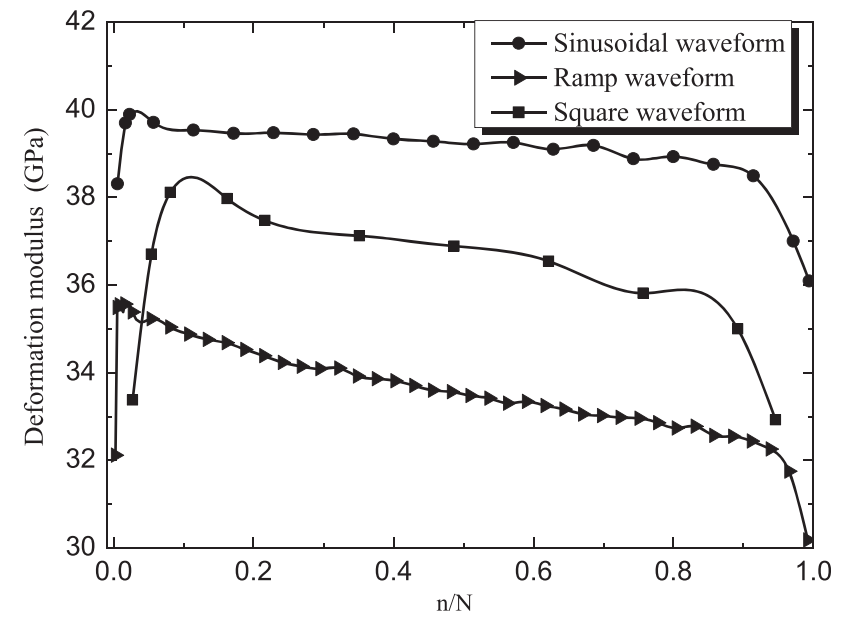

Figure 10. Relations of concrete deformation modulus and cyclic ratio under various loading waveforms.

with the three-phase process, which involves the increasing stage, slightly decreasing stage and accelerated decreasing stage. In the first increasing stage, micropores and microcracks are closed when loading is applied on the concrete, which makes the concrete structure much denser. The denser concrete presents linear elasticity. Hence the deformation modulus increases with the increasing of loading cycle ratio. In the second stage, the concrete shows fatigue deformation process. In the second stage, the deformation modulus slightly decreases with the cycle ratio; this stage accounts for the majority of the whole fatigue loading process. The third stage is the accelerated decreasing process of deformation modulus, which finally leads to the failure of concrete.

\section{Conclusions}

In this paper, research experiments on fatigue behaviour of concrete under cyclic tensile loading are conducted using electro-hydraulic servo-controlled MTS 322. The effect of various loading waveforms (square, ramp and sinusoidal waveforms) on the fatigue behaviour of concrete is determined. The axial strain and dissipated energy per loading cycle, damage evolution and deformation modulus are studied in this paper. The following concluding remarks can be drawn.

1. The hysteresis in stress strain curves presents three stages of loose-intensive-loose. The three-stage process is most obviously followed with sinusoidal waveform.

2. The accumulation of axial strain of concrete under fatigue tensile loading can be considered as a three-stage process: initial increase stage, increase at a constant speed stage and accelerated increase stage. In this work, experimental results obtained with the ramp waveform follow the most obvious rule of three-stage evolution process. 
3. The damage variable also presents the three-stage evolution process for concrete under fatigue tensile loading. They are the initial damage stage, stable increase stage and accelerated damage stage. The three-stage damage evolution is more obviously followed with sinusoidal and ramp waveforms than with square waveform.

4. The area of hysteresis loop increases and then tends to a stable value with the increasing of loading cycle ratio with a subsequent sudden increase until failure. It is evident that the amount of dissipated energy changes at different stages of fatigue life. At the beginning and end of the fatigue life, the higher rate of dissipated energy could be attributed to the formation of cracks.

5. The changed rule of deformation modulus for concrete is consistent in three waveforms. The evolution rule presents increasing-slightly decreasing-accelerated decreasing stages.

\section{Acknowledgements}

The authors would like to acknowledge the financial support from the National Natural Science Foundation of China (Grant No. 51509078) and Natural Science Foundation of Jiangsu Province (Grant No. BK20150820) and the Fundamental Research Funds for the Central Universities (2016B06014) granted to the corresponding author Xudong Chen.

\section{References}

[1] Scholz C H and Koczynski T A 1979 Dilatancy anisotropy and the response of rock to large cyclic loads. J. Geol. Res. Solid Earth 84(B10): 5525-5534

[2] Akai K and Ohnishi Y 1983 Strength and deformation characteristics of soft sedimentary rock under repeated and creep loading. Proceedings of the 5th ISRM Congress, International Society for Rock Mechanics

[3] Ge X, Jiang Y, Lu Y, et al 2003 Testing study on fatigue deformation law of rock under cyclic loading. Chin. J. Rock Mech. Eng. 22(10): 1581-1585 (in Chinese)

[4] Zhang Y, Xu J, Yang H, et al 2011 Effect of confining pressure on evolution law of hysteresis loop of sandstone under cyclic loading. Chin. J. Rock Mech. Eng. 30(2): 320-326 (in Chinese)

[5] Tao Z and Mo H 1990 An experimental study and analysis of the behaviour of rock under cyclic loading. Int. J. Rock Mech. Mining Sci. Geomech. Abstr. 27(1): 51-56

[6] Bagde M N and Petroš V 2005 Waveform effect on fatigue properties of intact sandstone in uniaxial cyclical loading. Rock Mech. Rock Eng. 38(3): 169-196

[7] Gong M and Smith I 2003 Effect of waveform and loading sequence on low-cycle compressive fatigue life of spruce. $J$. Mater. Civil Eng. 15(1): 93-99.

[8] Xiao J Q, Ding D X, Xu G, et al 2008 Waveform effect on quasi-dynamic loading condition and the mechanical properties of brittle materials. Int. J. Rock Mech. Mining Sci. 45(4): 621-626

[9] Medeiros A, Zhang X, Ruiz G, Yu R and Velasco M 2015 Effect of the loading frequency on the compressive fatigue behaviour of plain and fiber reinforced concrete. Int. J. Fatigue 70: 342-350

[10] Saucedo L, Rena C Y, Medeiros A, Zhang X and Ruiz G 2013 A probabilistic fatigue model based on the initial distribution to consider frequency effect in plain and fiber reinforced concrete. Int. J. Fatigue 48: 308-318

[11] Kesler C E 1953 Effect of speed of testing on flexural fatigue strength of plain concrete. Highway Res. Board Proc. 32: 251-258

[12] Raithby K D and Galloway J W 1974 Effects of moisture condition age, and rate of loading on fatigue of plain concrete. Special Publication 41 (Detroit, MI: American Concrete Institute) pp. 15-35

[13] Darter M I 1977 Development of design procedures. In: Design of a zero-maintenance plain jointed concrete pavement, vol. 1

[14] Murdock J W and Kesler C E 1958 Effect of range of stress on fatigue strength of plain concrete beams. J. Proc. 30(2): 221-231

[15] Tepfers R and Kutti T 1979 Fatigue strength of plain, ordinary, and lightweight concrete. ACI J. Proc. 76(5): 635-652

[16] Oh B H 1986 Fatigue analysis of plain concrete in flexure. $J$. Struct. Eng. 112(2): 273-288

[17] Shi X P, Fwa T F and Tan S A 1993 Flexural fatigue strength of plain concrete. ACI Mater. J. 90(5): 435-440

[18] Singh S P 2011 Fatigue strength of hybrid steel-polypropylene fibrous concrete beams in flexure. Proc. Eng. 14: 2446-2452

[19] Tepfers R, Sjöström G O, Svensson J I, et al 2011 Development of a method for measuring destruction energy and generated heat at fatigue of concrete. Proceedings of the $3 \mathrm{rd}$ International Conference on Civil Engineering, vol. 11, pp. 117-124

[20] Aramoon E 2014 Flexural fatigue behavior of fiber-reinforced concrete based on dissipated energy modeling. $\mathrm{PhD}$ Dissertation, University of Maryland

[21] Murthy A R C, Palani G S, Iyer N R, et al 2013 Residual strength evaluation of concrete structural components under fatigue loading. Sadhana 37(1): 133-147

[22] Chen X, Wu S and Zhou J 2013 Experimental study and analytical formulation of mechanical behavior of concrete. Constr. Build. Mater. 47: 662-670

[23] Chen X, Wu S, Zhou J, et al 2012 Effect of testing method and strain rate on stress-strain behavior of concrete. $J$. Mater. Civil Eng. 25(11): 1752-1761

[24] Chen X, Wu S and Zhou J 2014 Quantification of dynamic tensile behavior of cement-based materials. Constr. Build. Mater. 51: 15-23

[25] Chen X, Wu S and Zhou J 2013 Experimental study on dynamic tensile strength of cement mortar using split Hopkinson pressure bar technique. J. Mater. Civil Eng. 26(6): 04014005

[26] Chen X, Wu S and Zhou J 2013 Strength values of cementitious materials in bending and tension test methods. $J$. Mater. Civil Eng. 26(3): 484-490

[27] Guo Y, Zhao K, Sun G, et al 2011 Experimental study of fatigue deformation and damage characteristics of salt rock under cyclic loading. J. Rock Soil Mech. 32(5): 1353-1359 (in Chinese) 